\title{
Hydrodynamics of Supernova Evolution in the Winds of Massive Stars
}

\author{
Vikram V. Dwarkadas \\ Astronomy and Astrophysics, Univ of Chicago, 5640 S Ellis Ave AAC 010c, Chicago IL \\ 60637 \\ vikram@oddjob.uchicago.edu
}

\begin{abstract}
Core-Collapse supernovae arise from stars greater than $8 M_{\odot}$. These stars lose a considerable amount of mass during their lifetime, which accumulates around the star forming wind-blown bubbles. Upon the death of the star in a spectacular explosion, the resulting SN shock wave will interact with this modified medium. We study the evolution of the shock wave, and investigate the properties of this interaction. We concentrate on the evolution of the SN shock wave in the medium around a 35 solar mass star. We discuss the hydrodynamics of the resulting interaction, the formation and growth of instabilities, and deviations from sphericity.
\end{abstract}

Subject headings: supernova remnants; hydrodynamics; instabilities; stellar winds; massive stars; wind-blown bubbles; shock waves

\section{INTRODUCTION}

Mass-loss from stars is a ubiquitous process. Massive stars $\left(>8 M_{\odot}\right)$ lose a considerable amount of mass before they explode. This material collects around the star, forming a circumstellar (CS) wind-blown bubble. At the end of its life, the star will explode in a cataclysmic supernova (SN) explosion, and the resulting shock wave will interact with this medium. The further evolution of the resulting supernova remnant will depend on the properties of this medium.

In this paper we discuss the evolution of the surrounding medium around massive stars, and the subsequent interaction of the SN shock wave with this medium following the star's death. The rich and complex dynamics of the various interactions leads to the formation and growth of a variety of hydrodynamic instabilities, which we will focus on in this paper. It 
may be possible to simulate some of these hydrodynamic situations with available laboratory apparatus, and we hope that this work will further stimulate laboratory experiments of realistic astrophysical phenomena, particularly those involving radiative shocks.

\section{SN-Circumstellar Interaction}

It has been realized over the years that the medium around a core-collapse SN is continually being sculpted during the progenitor star's lifetime, by the action of winds and outbursts. Chevalier \& Liang (1989) discussed the interaction between a SN shock wave and the surrounding wind-blown bubble formed by the pre-SN star. However analytic arguments can only be extended so far, and numerical simulations are required to study the subsequent non-linear behavior. A series of papers in the early 90's (Tenorio-Tagle et al. 1990, 1991; Rozyczka et al. 1993) explored some aspects of this. Since then our observational knowledge of this phenomena has multiplied exponentially, thanks to the availability of space based data in the optical, X-ray and infrared bands, and the stream of data pouring in from observations of SN 1987A. The latter has become the poster-child for SN evolution in wind-blown bubbles, having shaped and confirmed many of our views.

The basic details of SN interaction with wind-blown bubbles were outlined in the papers listed above, and further elaborated on by Dwarkadas (2005). The mass loss results in the formation of a circumstellar (CS) wind blown cavity surrounding the star, bordered by a thin, dense, cold shell. The typical structure of this wind-blown bubble for constant wind properties (Weaver et al. 1977) is shown in Figure 1, and consists of an outwards expanding shock wave $\left(\mathrm{R}_{o}\right)$, and a wind termination shock $\left(\mathrm{R}_{t}\right)$ that expands inwards in a Lagrangian sense, separated by a contact discontinuity $\left(\mathrm{R}_{c d}\right)$. In general most of the volume between $\mathrm{R}_{t}$ and $\mathrm{R}_{c d}$ is occupied by a low-density, high pressure shocked wind bubble, surrounded by the extremely dense shell. Most of the mass is contained in the dense shell. When the SN shock wave interacts with this bubble, it quickly finds itself in a medium with density much lower than that of the ISM. Consequently, the emission from the remnant, which arises mainly from CS interaction (Chevalier \& Fransson 1994), will be considerably reduced compared to evolution within the ISM.

It comes as no surprise then that the subsequent evolution depends primarily on a single parameter $\Lambda$, the ratio of the mass of the dense shell to that of the ejected material. For very small values $\Lambda \ll 1$ the effect of the shell is negligible as expected. For values of $\Lambda \lesssim 1$ interaction with the shell results in considerable deceleration of the SN shock wave. The X-ray luminosity can increase by orders of magnitude upon shock-shell collision. A transmitted shock wave enters the shell, while a reflected shock wave moves back into the 

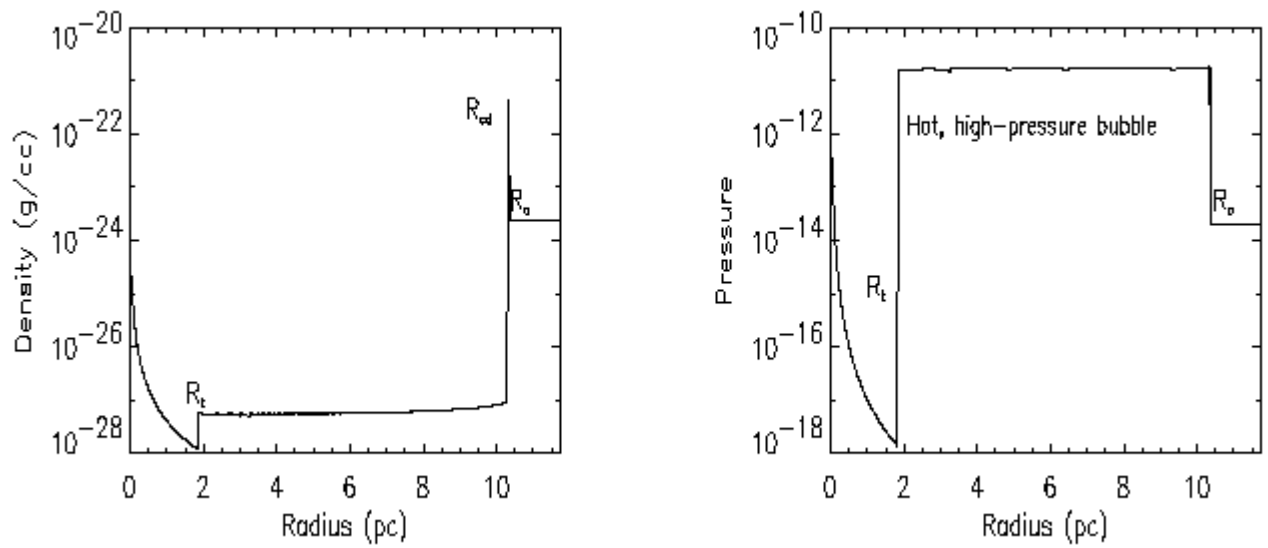

Fig. 1.- Density and pressure profiles for a circumstellar wind-blown bubble

ejecta. If X-ray images were taken just after the interaction, they would show the presence of a double-shelled structure as the reflected shock begins to move inwards. In about 10 doubling times of the radius the SN begins to 'forget' about the existence of the shell. The remnant density profile changes to reflect this, and consequently the X-ray emission from the remnant, which depends on the density structure, will also change. The reflected shock will move to the center and presumably be reflected back, while the transmitted shock will slowly exit the shell and eventually separate from it.

As the ratio $\Lambda$ increases, more of the kinetic energy from the remnant is converted to thermal energy of the shell. The transmitted shock is considerably slowed down, and in extreme cases $(\Lambda \gg 1)$ may even be trapped in the shell. The high pressure behind the reflected shock will impart a large velocity to the shock, and therefore thermalization of the ejecta is achieved in a much shorter time as compared to thermalization by the SN reverse shock. Upon reaching the center the reflected shock bounces back, sending a weaker shock wave that will collide with the shell. In time a series of shock waves and rarefaction waves are seen traversing the ejecta. Each time a shock wave collides with the dense shell a corresponding (but successively weaker) rise in the X-ray emission from the remnant is seen.

We have outlined the basics of SN interaction with CS wind-blown shells. One-dimensional models are fully described in Dwarkadas (2005). We wish to present herein results from multi-dimensional models. 


\section{CS Medium around a $35 M_{\odot}$ Star}

The above description considered an idealized wind-blown bubble formed by the interaction of a fast wind with the surrounding medium, where the properties of both are constant in time. In reality, as a massive star evolves, the wind properties change with time. In particular after a star leaves the main sequence, its mass-loss properties change considerably. This will give rise to a much more complicated bubble structure than is shown in Figure 1.

In order to explore more realistically the medium surrounding a core-collapse $\mathrm{SN}$, we have taken stellar evolution calculations from several groups, and investigated the evolution of the surrounding medium as the star evolves. In this paper we discuss the evolution of the medium around a $35 M_{\odot}$ star, from an evolutionary model provided to us by Norbert Langer. The star begins its life on the main sequence as an O star, then expands to become a Red Supergiant (RSG), and finally ends its life as a Wolf-Rayet (WR) star. The mass-loss rate and wind velocity over the evolution are shown in Figure 2. During the main-sequence stage, the mass-loss rate is a few times $10^{-7} M_{\odot} / \mathrm{yr}$, and the wind velocity is about $3000-4000 \mathrm{~km} / \mathrm{s}$.

Once the star swells to become a red supergiant, the wind velocity reduces by more than 2 orders of magnitude, and the mass-loss rate increases to almost $10^{-4} M_{\odot} / \mathrm{yr}$. The WR wind shows a slight drop in mass-loss rates by a factor of a few from the RSG phase, but a steep increase in wind velocity by two orders of magnitude. We use these values as input boundary values at each timestep to our code, which then computes the structure of the nebula over time. Unlike a previous computation (Garcia-Segura et al. 1996) our computation is fully two-dimensional right from the start. We use a grid consisting of 600 zones in both the radial and azimuthal directions. The code used is the $\mathrm{VH}-1$ numerical hydrodynamics code, a multi-dimensional code that solves the equations of fluid dynamics on a Lagrangian grid, and then remaps them onto an Eulerian grid. Radiative cooling is included via a cooling function, but we have not included the effects of ionization. A grid that expands outwards with the outgoing shock wave is used, although no new zones are added, i.e. the grid is not adaptive. Initially the wind occupies about 20 zones on the grid, depending on the grid resolution. The mass-loss rate and velocity of the wind are used to compute the density and velocity of the inflow, which are used as the input boundary conditions at each timestep. The initial setup is uniform and no perturbations are applied to the system. Perturbations that arise are due to effects such as non-spherical shocks on the spherical grid.

The evolution of the medium is shown in Figure 3. In the main sequence stage (3a, $3 \mathrm{~b}$ ), although the wind properties are changing continuously, the nebular structure is not very different from that expected from the idealized, two-wind case of a fast wind interacting with a slower wind, both of which have constant wind properties. A thin shell of swept-up material is formed, and the volume of the nebula is mostly occupied by a hot, low density 

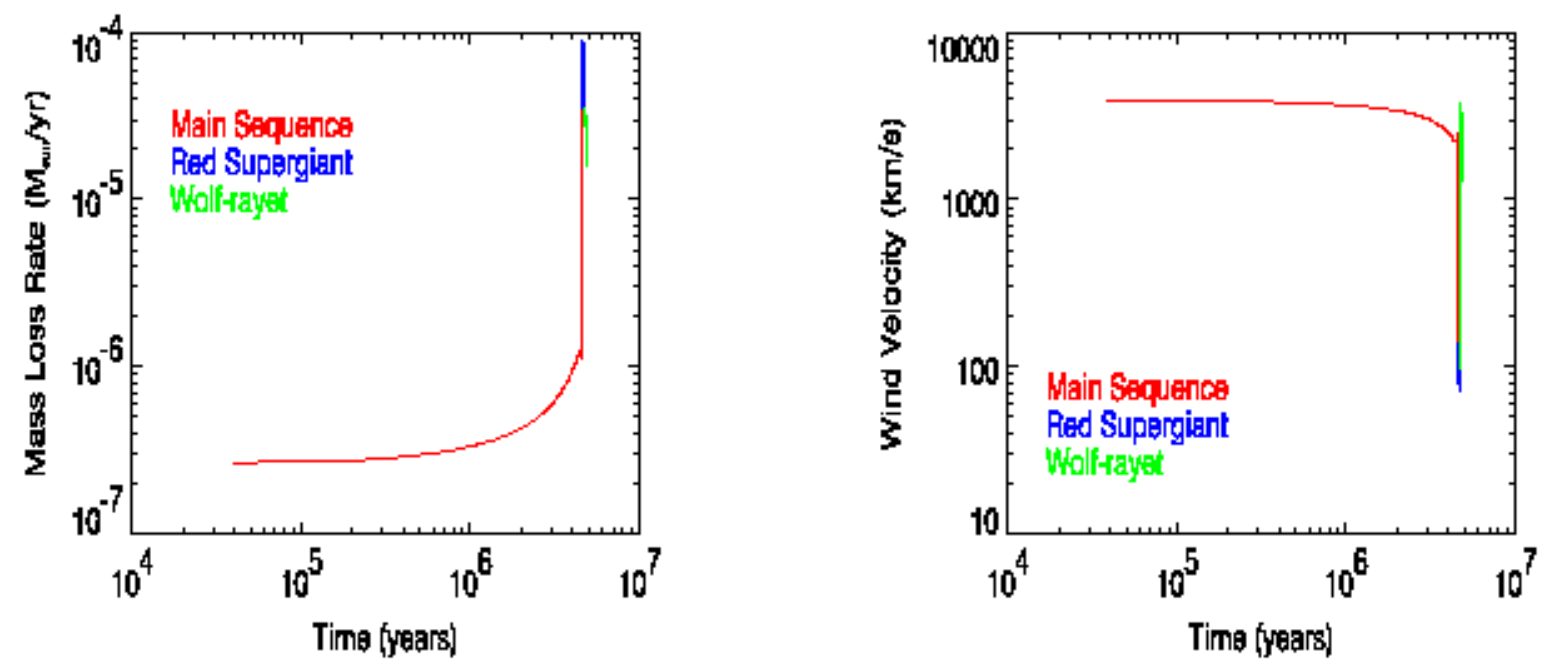

Fig. 2.- Evolution of the mass-loss rate (left) and wind velocity (right) for the $35 M_{\odot}$ star during its lifetime

bubble. The shell is on the whole mostly stable, although it shows some wrinkles. These arise mainly from shearing, due to flow of gas along the contact discontinuity. However these instabilities are not highly pronounced, and do not appear to grow to any significant extent. In lower-resolution simulations presented earlier (Dwarkadas 2001, 2004) we had suggested that the shell is unstable to some type of thin-shell instability (Vishniac 1983). We have seen the same instability in simulations of the medium around a $40 M_{\odot}$ star. The higher resolution calculations presented here do not show a strong presence of such an instability however. This is a topic still under investigation.

The interior of the nebula shows significant fluctuations in density and pressure, and vortices are visible in the velocity flow. Since the mass-loss rate and wind velocity are changing at every timestep, the position of the reverse shock is not fixed on the grid, but moves slightly every timestep with respect to the outer shock. The changing position of the reverse shock from one timestep to another results in the deposition of vorticity into the shocked wind, which is then carried out with the shocked flow. This results in an interior that is quite inhomogeneous, with significant density fluctuations.

When the star leaves the main sequence and becomes a RSG star, its radius increases considerably, the wind velocity $\left(V_{\text {wind }}\right)$ drops by two orders of magnitude, while the massloss rate $(\dot{M})$ increases appropriately. Thus the wind density, proportional to $\dot{M} / V_{\text {wind }}$, goes up by several orders of magnitude. A new pressure equilibrium is established, and a shock 
front is formed in between the RSG wind and main sequence bubble as the RSG wind is decelerated by the bubble pressure. The RSG wind piles up against this shock, forming a thin dense shell of RSG material. No hot, low-density cavity is present. The shell decelerates as it expands outwards, satisfying the classic case of Rayleigh-Taylor (R-T) instability, and Rayleigh-Taylor fingers are seen expanding outwards from the high-density shell into the lowdensity ambient medium (3c). Some of the filaments show the presence of sub-filaments growing from the main one, and the expanded heads of many of the filaments are a sign of Kelvin-Helmholtz instabilities resulting from the shear flow in between the filaments and the surrounding medium. Unfortunately, since we need to resolve the entire bubble, the resolution is not large enough to study the growth of the fingers in detail.

The star leaves the RSG phase and loses its outer hydrogen envelope, becoming a WR star in the process. The compact star now gives off a very fast wind, not unlike in the $\mathrm{O}$ star stage, but with a mass-loss rate that is much higher than in the main sequence, and just a few times lower than the RSG stage. The supersonic WR wind creates a wind-blown bubble in the RSG wind. The dense W-R shell is accelerated by the high pressure, low density interior as it expands outwards, leading to the triggering of the Rayleigh-Taylor instability (3d). In this case R-T fingers are seen expanding inwards from the dense shell into the low-density cavity. The large momentum of the WR shell causes the RSG shell to fragment, and carries the material outwards (3e), speeding up as it enters the low-density bubble. Due to the fact that the WR wind is carrying fragments of the RSG material, and that it travels through a medium with considerable fluctuations in density and pressure, its expansion is not completely spherical. The collision of this slightly aspherical wind with the main sequence shell gives rise to a reflected shock that moves back into the bubble. The asphericity is accentuated in the reflected shock, which moves inwards, before finally coming to rest in a wind-termination shock where the ram pressure of the freely expanding wind is equal to the thermal pressure within the bubble. The wind-termination shock when it forms is consequently also not spherical but slightly elongated along the equator (3f). As we shall show later this has important consequences for the expansion of the SN shock wave.

\section{SN-CSM interaction in the case of the $35 M_{\odot}$ star}

At the end of the WR stage, the stellar mass remaining is $9.1 M_{\odot}$. We assume that

the star then explodes in a SN explosion. A remnant of $1.4 M_{\odot}$ is left behind, and the remaining mass is ejected in the explosion. We use the prescription of Chevalier \& Fransson (1994) to describe the ejecta structure as a power-law with density, with power-law index of 7 . We compute the evolution of the SN described by this density profile expanding into 
the unshocked wind, and then map it onto the grid containing the bubble simulation. This calculation was also carried out using 600 X 600 zones.

The evolution of the SN shock wave is shown in Figure 4. It starts out as expected, with the formation of a forward and reverse shock structure (Fig 4a). The interaction of the spherical forward shock with the aspherical wind termination shock, susceptible to the Richtmeyer-Meshkov instability, reveals quite interesting dynamics. Since the SN shock is spherical while the wind termination shock is slightly more elongated towards the equator, the interaction first takes place close to the symmetry axis. A transmitted shock moves out into the shocked bubble, while a reflected shock moves back. Different parts of the SN shock collide with the wind-termination shock at different times, leading to transmitted shocks with a small but non-negligible velocity spread (Fig 4b). The composite transmitted shock then expands in the inhomogeneous medium, interacting with several large density fluctuations on the way. The net result is a very corrugated shock wave that expands outwards towards the main sequence shell (Fig 4c). The wrinkles are similarly prevalent in the reverse shock also. The wrinkled shock collides in a piecemeal fashion with the main sequence shell, with some parts of the shock colliding before others (Fig 4d, 4e). Each collision with the shell will give rise to an increase in the optical and X-ray emission at that point. Therefore some parts of the shell will brighten before others. It is interesting to note that a similar phenomenon has been observed in SN 1987A, where bright spots appear successively around different parts of the equatorial ring (Sugerman et al. 2002)

As each portion of the forward shock wave collides with the shell, a reflected and transmitted shock pair is formed. The shell is dense enough that the transmitted shock does not emerge from the shell for a long period. The reflected shock meanwhile travels back towards the origin. However, as seen in Figure 4, the velocity of each piece differs considerably from the next, both in magnitude as well as direction. The shape of the reflected shock therefore deviates significantly from spherical, and some parts of the reflected shock reach the symmetry axis before the rest has traveled far into the interior (Figure 4f). This gives the remnant a very asymmetric shape, and results in some portions of the ejecta being much hotter than others.

\section{Conclusions}

The surroundings of massive stars are shaped by the mass-loss from the progenitor

star. This can lead to a complicated density structure for the surrounding medium, the formation and growth of various hydrodynamical instabilities, deposition of vorticity and onset of turbulence. When the star explodes as a SN remnant, the SN shock wave will 
interact with this ambient medium. The inhomogeneous structure of the ambient medium can cause distortions in the SN shock wave as it expands outwards, which are magnified by the turbulence in the wind-blown structure. In this paper we have shown that the end result can be a wrinkled shock wave whose impact with the surrounding shell occurs in a piecemeal fashion. As each part of the shock wave hits the shell, it will brighten up in the optical and X-ray regime, a phenomenon that is observable in SN 1987A. We do caution that this comparison is illustrative only. Our numerical models are not meant to simulate SN 1987A, whose progenitor star was a much lower mass B3Ia star.

Our simulations show that the complicated structure of the medium may result in deviations from spherical symmetry for the SN shock wave. Even though the expansion starts out as spherical, the final shape of the remnant may deviate considerably from sphericity. Most of the emission from the remnant arises from the high pressure region in between the forward and reverse shocks. The distorted shape of this emitting region is clearly visible in Figure 4, and this will be reflected in observations of the remnant.

Herein we have summarized the features of multi-dimensional models of SN evolution in the environments shaped by massive stars. Further details are available from Dwarkadas (2006).

Vikram Dwarkadas is supported by award \# AST-0319261 from the National Science Foundation, and by NASA through grant \# HST-AR-10649 from STScI. We thank the anonymous referee for suggestions that helped to improve this paper. We acknowledge useful discussions with Roger Chevalier which were particularly helpful in identifying the various instabilities that were observed. We are grateful for comments from John Blondin and Thierry Foglizzo. This research was supported in part by the National Science Foundation under Grant No. PHY99-07949 to the KITP.

\section{REFERENCES}

Chevalier, R. A. \& Fransson, C. 1994, ApJ, 420, 268

Chevalier, R. A. \& Liang, E. P. 1989, ApJ, 344, 332

Dwarkadas, V. V, 2006, to be submitted to ApJ

Dwarkadas, V. V, 2005, ApJ, 630, 892

Dwarkadas, V. V, 2004, in Cosmic explosions in three dimensions : asymmetries in supernovae and gamma-ray bursts. Eds P. Hoflich, P. Kumar \& J. C. Wheeler, (Cambridge: CUP), 74 
Dwarkadas, V. V, 2001, JKAS, 34, 243

Garcia-Segura, G., Langer, N., \& MacLow, M.-M. 1996, A\&A, 316, 133

Rozyczka, M., Tenorio-Tagle, G., Franco, J., Bodenheimer, P. 1993, MNRAS, 261, 674

Sugerman, B., E., K, et al. 2002, ApJ, 572, 209

Tenorio-Tagle, G., Rozyczka, M., Franco, J., \& Bodenheimer, P. 1991, MNRAS, 251, 318

Tenorio-Tagle, G., Bodenheimer, P., Franco, J., \& Rozyczka, M. 1990, MNRAS, 244, 563

Vishniac, E. T. 1994, ApJ, 428, 186

Vishniac, E. T. 1983, ApJ, 274, 152

Weaver, R., McCray, R., Castor, J., Shapiro, P., \& Moore, R. 1977, ApJ, 218, 377 

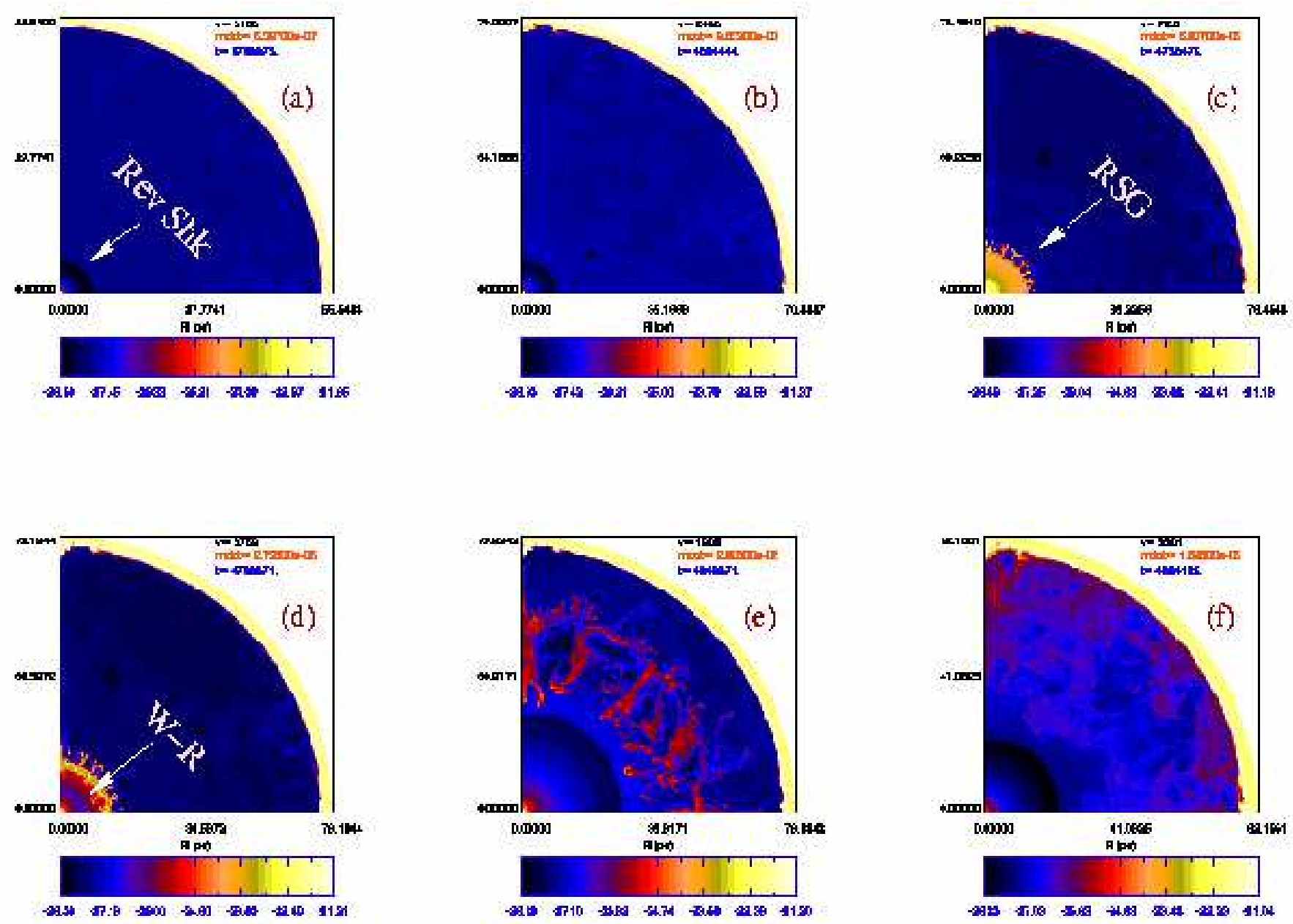

Fig. 3.- Density evolution of the medium a round a $35 M_{\odot}$ star with time. The wind properties at each stage are given in the top right hand corner of each panel. The velocity is in $\mathrm{km} / \mathrm{s}$, the mass loss rate in $M_{\odot} / \mathrm{yr}$, and the time in years. The color bar shows the logarithm of the gas density in units of $\mathrm{g} \mathrm{cc}^{-1}$ 

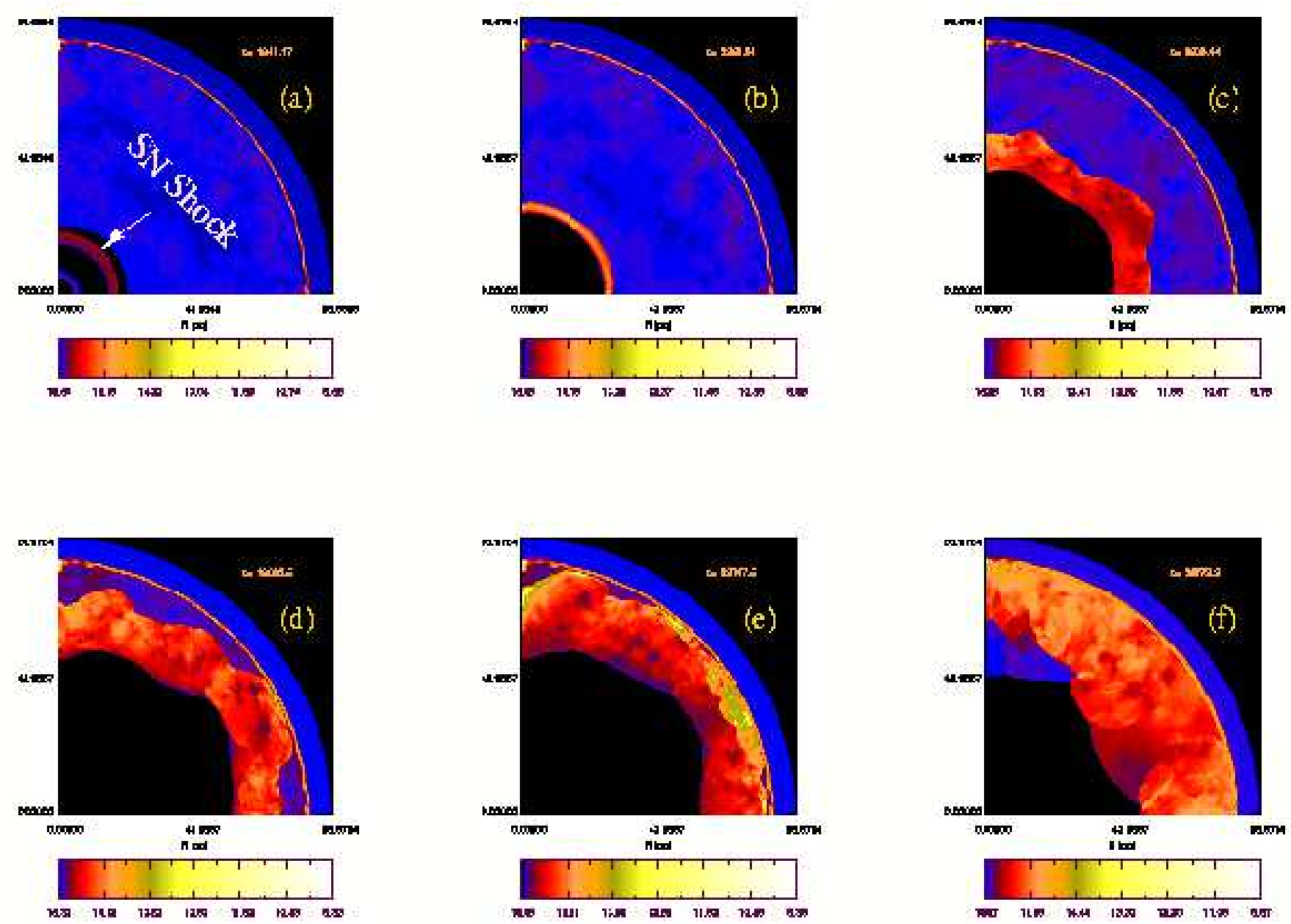

Fig. 4. - Pressure evolution of the SN shock wave within the WR bubble 